\title{
Refining the interface between automation and crystallisation
}

\author{
$\underline{\text { Janet Newman }}{ }^{1}$, Bevan Marshall ${ }^{1}$, Nicolas Rosa ${ }^{1}$, Marko Ristic ${ }^{1}$, Christopher J. Watkins ${ }^{2}$
}

1. Biomedical Manufacturing CSIRO Parkville VIC Australia

2. Scientific Computing CSIRO Clayton VIC Australia

The state of the art in macromolecular crystallisation is generally a trial and error approach to finding a suitable mixture of chemicals and an appropriate geometry (e.g. sitting drop experiments at 20 degrees) that will support crystal growth. Although there are a number of commercially available tools for easing the toil of macromolecular crystallisation, what is generally lacking in most labs is a smooth workflow which incorporates the commercial solutions.

In the Collaborative Crystallisation Centre (C3), which is a crystallisation technology platform setting up 2000 experiments annually, we have built up a number of custom applications to smooth the flow of information within the lab. These include software tools for booking resources, creating experiments and interpreting results. An overview of the process will be presented. In particular we will discuss some of the applications developed for QA/QC, for example, one that checks if drops have been dispensed as expected. We will also present the tools that we have developed which allow users to observe, score and optimise their crystallisation experiments. Many of our tools have been developed as open source applications, and could be easily implemented in other laboratories. 